\title{
Investigating the Effect of Roughness and Stiffness in 2D Aeroelastic Around Oscillatory Airfoil
}

\author{
M.R. SABER *, M.H. DJAVARESHKIAN** \\ *Department of Mechanical Engineering, Faculty of Engineering, Ferdowsi University of Mashhad, Mashhad, IRAN
}

\begin{abstract}
${ }^{\ddagger}$ Corresponding Author: Mechanical Engineering Department, Faculty of Engineering, Ferdowsi University of Mashhad Mashhad, Iran M.H. Djavareshkian, IRAN
\end{abstract}

\begin{abstract}
In this research, a software has been developed to investigate the effect of roughness and stiffness in twodimensional aeroelastic in unsteady viscous flow around oscillatory airfoil. In this simulation to solve the Navier-Stokes equations, finite volume method has been used in the code with a high resolution scheme for fluid and structure simulation in transonic flows. For this purpose, fluid and structural behavior is solved separately at each time step and the effect of each one on the other is considered. For computing convection term in transonic unsteady compressible flow, high order SBIC (Second and Blending Interpolation Combined) scheme based on discretization of Normalized Variables Diagram (NVD) is used. Here the technique of inlet velocity vector oscillation which is a simpler method in comparison with rather complicated methods such as dynamic mesh is applied. The two-dimensional motion equations are obtained from the Lagrangian equations which are combined with the aerodynamic equations. The results of validation show that the extracted data has a desirable accuracy and had good agreement to experimental data. The FSI results show that, 1: Lift coefficient in smooth surface is more than the rough surface and also the drag coefficient in rough surface is more than the smooth surface, 2: Shock strength is weaker in the rough surface, 3: The shock's place has moved to leading edge in the rough surface, 4: The number of oscillations in rough surface is reduced, 5:The structural stability of the airfoil when the surface of the airfoil is rough is much greater than smooth surface, 6: Because the density of the air and the amplitude of the oscillations are small and also small effect on the lift and drag coefficients, can be ignored the added mass in this simulation method.
\end{abstract}

Keywords: FSI, Aeroelastic, Oscillation, Transonic, Roughness, Stiffness, Unsteady

Received: February 20, 2020. Revised: September 2, 2020. Accepted: September 17, 2020. Published: October 1, 2020.

\section{Introduction}

In recent years considerable attention has been paid to aeroelastic behaviors in various topics such as turbines, compressors and wing fighter aircraft. Nowadays linear aeroelastic constitutes a large part of aeroelastic studies but when the effects of roughness, shock waves, flow separation and viscosity effects are considered concurrently, a little research has been done. Particularly in the transonic flow non-linear parameters play an important role in the aeroelastic behavior which linear theories do not have the ability to study these behaviors accurately. One of the important problems in aeroelastic is the stability of the structure. By applying roughness to the airfoil surface, the distribution of the pressure and velocity on airfoil has changed and the structure is more stable.

Aerodynamic forces increase with increasing flow velocity, while elastic stiffness is independent of flow velocity, therefore the structure becomes unstable with speed of flow. This instability can cause finally lead to fail the structure. A significant phenomenon in aeroelasticity is flutter, in which fluctuations grow and produce great dynamical forces [1].At first a summary of the work done in the field of aeroelastic is presented then the motivation and purpose of this research are given.

Lapointe and Dumas made a numerical simulation of the pitch-plunge forced oscillation on the NACA0012 airfoil and showed that in harmonic motion the oscillation amplitude and the pitch angle were immortal and constant [2]. Mowat used nonlinear aeroelastic modeling by using and coupling fluid-structure interaction. They used the Euler equations in the fluid region and in the solid region discrete equations by using Reduced Order Model (ROM). Their results show the effects of nonlinear terms in the governing equations [3]. Khairil and Zorkipli simulated the aeroelastic system by using nonlinear aerodynamics. Their results show a good trend with the experimental data [4]. Unger investigated oscillating airfoil for flapping wing propulsion and their results had a good agreement with the data of wind tunnel [5]. Riso investigated the numerical aeroelastic analysis of a flat plate in arbitrary motion with high amplitude. In their research, fluid is assumed to be a non-viscous, incompressible and potential flow [6]. Medjroubi used the spectral element method in Reynolds between 800 and 10,000 to discrete the Navier Stokes equations. They showed that when the frequency and amplitude of the oscillation are constant, the Reynolds number does not have a big effect on flow field and aerodynamic forces. Also they showed that if the amplitude of the oscillation increases, it wills greatly effect on the wake and the aerodynamic coefficients [7]. Yuan studied numerically an uncompressible flow on airfoil NACA0012 in free oscillation and two degrees of freedom. They displayed that by increasing Reynolds, the number of the amplitude of the oscillation increases [8]. Banavara and Dimitrov proposed Reduced Order Model (ROM) in the aeroelastic behavior of the wings in the transonic flow because the linear aerodynamics theory did not have proper 
predictions of detached flow due to nonlinear terms [9]. Akkala and Eslampanah examined the aerodynamic behavior of a flexible airfoil in plunge's motion experimentally. They used PIV to determine the vortex and flow field. They showed that at low frequencies the flexible wing was under the slightest deformation and also the efficiency of flexible wing was higher than the rigid wing [10]. Razak studied aeroelastic behavior of a rectangular wing in pitch-plunge motion by using acceleration and pressure measurements in an experimental work. They showed that the phenomenon of stall flutter and flutter are related to the frequency of oscillations [11]. Walker and Patil examined the flexibilities in the form of Chebyshev polynomials. They used the expansion of unsteady thin airfoil theory for aerodynamics section that was developed by the odersen for a rigid body [12]. Williams proposed a method for linearizing the unsteady transonic flow that included the shock waves [13]. Taobai studied the effect of surface roughness on the turbine blades. The results show that at a rough surface, the velocity in the boundary layer is greater than smooth surface, and at low Reynolds the aerodynamic loss is lowered [14]. Bouhelal studied roughness on the aerodynamic performance of horizontal axial wind turbine blades. He was used a modification of the universal standard wall function. The results show that by increasing roughness height the intensity of turbulence near the wall increases [15]. Ecesagol and Marcelo studied roughness issues on wind turbine blades. They provided solutions to reduce roughness effects to increase power production [16]. Beatriz and Arturo studied the roughness distribution on the wind turbine airfoils. Their results showed that by applying surface roughness, the lift coefficient decreased and the drag coefficient increased [17].

In this research, a simple, accurate, and time-saving way to simulate aeroelastic transience on the airfoil has been developed. Two-dimensional software has been developed to simulate transonic unsteady compressible flow passing an oscillatory airfoil and used a numerical finite volume method with a pressure base algorithm. To calculate convection terms high order SBIC scheme is used and the technique oscillation of flow boundary condition method is applied which is simpler method in comparison with other such as dynamic mesh. To simulate Navier Stokes equations a modified $\boldsymbol{\kappa}-\boldsymbol{\varepsilon}$ model for compressible flow is exerted. In this method the Normalized Variable Diagram (NVD) scheme has been used. The two-dimensional motion equations are obtained from the Lagrangian equations which are combined with the aerodynamic equations. This research sets out to study nonlinear aerodynamic effects on aeroelastic behavior of self-sustained oscillating and forced oscillation of rigid airfoil. For this purpose the structural stiffness and roughness and other effective parameters have been investigated.

\section{Governing Equations and Discretization}

\subsection{Fluid Equations}

The governing equations in this simulation include the equation of continuity, momentum equations and the energy equation.

$$
\frac{\partial \rho}{\partial t}+\nabla \cdot\left(\rho v_{i}\right)=0
$$

$$
\begin{aligned}
& \frac{\partial\left(\rho v_{i}\right)}{\partial t}+\frac{\partial\left(\rho v_{i} v_{j-T}\right)}{\partial x_{j}}=S_{i}^{v} \\
& \frac{\partial(\rho \Phi)}{\partial t}+\frac{\partial\left(\rho v_{j} \Phi-q_{j}\right)}{\partial x_{j}}=S^{\Phi} \\
& T_{i j}=\mu\left(\frac{\partial v_{i}}{\partial x_{j}}+\frac{\partial v_{j}}{\partial x_{i}}\right)-\frac{2}{3} \mu \frac{\partial v_{n}}{\partial x_{n}} \delta_{i j}-P \delta_{i j} \\
& q_{j}=\Gamma_{\Phi}\left(\frac{\partial \Phi}{\partial x_{j}}\right)
\end{aligned}
$$

These equations are solved in a pressure-based algorithm by PISO algorithm. The turbulence model used in this simulation is a modified $\boldsymbol{\kappa}-\boldsymbol{\varepsilon}$ model [18].

$$
\begin{aligned}
& \quad \frac{\partial}{\partial t}(\rho k)+\frac{\partial}{\partial x_{j}}\left(\rho v_{j} k-\Gamma_{k} \frac{\partial k}{\partial x_{j}}\right)=D_{\text {comp }}+G-\rho \varepsilon+ \\
& \Theta_{\text {diff }} \\
& \quad \frac{\partial}{\partial t}(\rho \varepsilon)+\frac{\partial}{\partial x_{j}}\left(\rho v_{j} \varepsilon-\Gamma_{\varepsilon} \frac{\partial \varepsilon}{\partial x_{j}}\right)=c_{1} \frac{\varepsilon}{k} G-c_{2} \rho \frac{\varepsilon^{2}}{k} \\
& G=\mu_{t}\left(\frac{\partial v_{i}}{\partial x_{j}}+\frac{\partial v_{j}}{\partial x_{i}}\right) \frac{\partial v_{i}}{\partial x_{j}}-\frac{2}{3} \mu_{t} \delta_{i j}\left(\frac{\partial v_{n}}{\partial x_{n}}+\rho k\right) \frac{\partial v_{i}}{\partial x_{j}}
\end{aligned}
$$$$
\Theta_{\text {diff }}
$$

Here, $D_{\text {comp }}$ and $\Theta_{\text {diff }}$ are the effects of compressibility in equations [19] and also the models proposed by Yang, are adopted [14].

$$
\begin{aligned}
& \Theta_{\text {diff }}=0.0 \\
& D_{\text {comp }}=-\frac{\mu_{t}}{\rho^{2}} \frac{\partial \rho}{\partial x_{i}} \frac{\partial P}{\partial x_{i}}-\frac{9}{55} \rho k \frac{\partial v_{i}}{\partial x_{i}}
\end{aligned}
$$

The constant coefficients in the above equations are given in Table 1.

Table1.Values of coefficients in turbulence model

\begin{tabular}{ccccc}
\hline$c_{\mu}$ & $\sigma_{k}$ & $\sigma_{\varepsilon}$ & $c_{1}$ & $c_{2}$ \\
\hline 0.09 & 1.0 & 1.3 & 1.44 & 1.92 \\
\hline
\end{tabular}

To investigate the effect of roughness on the surface in equations, the wall functions are used.

$$
\begin{aligned}
& u^{+}=\frac{u}{u_{\tau}}=\frac{1}{\kappa} \ln \left(\mathrm{E} y^{+}\right)+\beta-\Delta \beta \\
& u_{\tau}=\sqrt{\frac{\tau_{w}}{\rho}} \\
& y^{+}=\frac{\rho y_{p} \sqrt{\frac{\tau_{w}}{\rho}}}{\mu} \\
& \Delta \beta=\frac{1}{\kappa} \ln \left(1+0.3 h_{s}^{+}\right) \\
& h_{s}^{+}=\frac{h_{s} u_{\tau}}{\vartheta} \\
& \left\{\begin{array}{lr}
0<h_{s}^{+}<5 & \text { smooth } \\
5<h_{s}^{+}<70 & \text { transitional roughness } \\
70<h_{s}^{+} & \text {fully rough }
\end{array}\right.
\end{aligned}
$$

Where $y_{p}$ is distance to the wall and $\mathrm{E}=9.793$ is empirical constant. As can be seen $\Delta \beta$ has a relation with roughness height and in different references for different conditions, the coefficients used in these equations are different.

\subsubsection{Discretization}

The finite volume method is used to discrete equations Fig.1. For this purpose the Gaussian theorem has been used. For any variable $\varnothing$ the result of the integration yield is: 


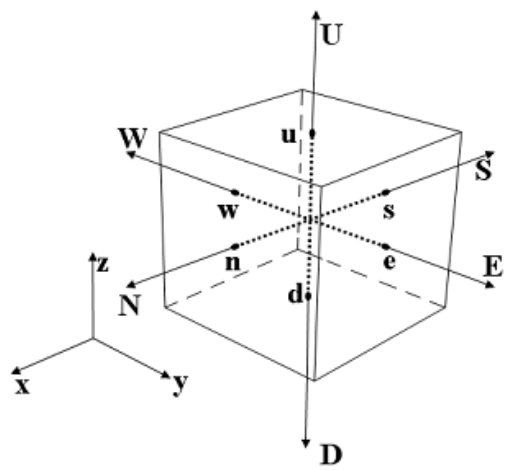

Fig1. Finite volume and storage arrangement

$\frac{\delta v}{\delta t}\left[(\rho \phi)_{p}^{n+1}-(\rho \phi)_{p}^{n}\right]+I_{e}-I_{w}+I_{n}-I_{s}=S_{\phi} \delta v$

The diffusion flux is approximated by central differences. A representation of the convective flux for cellface $w$ is:

$$
I_{w}=F_{w} \Phi_{w}=\Phi_{w}(\rho V A)_{w}
$$

To determine the value of $\Phi_{w}$ must interpolate between the neighboring points of the cellthat is defined by the SBIC scheme that is based on the NVD technique. The $\Phi_{\mathrm{w}}$ value is obtained under the following conditions from the SBIC scheme.

$$
\begin{aligned}
& \Phi_{w}=\Phi_{p} \\
& \text { If } \Phi_{p} \notin[0,1] \\
& \Phi_{w}=\left(1+\frac{x_{p}-x_{w}}{k\left(x_{p}-1\right)}\right) \Phi_{p}-\frac{x_{p}-x_{w}}{k\left(x_{p}-1\right)} \Phi_{p}{ }^{2} \text { If } \Phi_{p} \in[0, k] \\
& \Phi_{w}=\frac{x_{w}-1}{x_{p}-1} \Phi_{p}+\frac{x_{p}-x_{w}}{x_{p}-1} \quad 0 \leq \mathrm{k} \leq 0.5 \quad \text { If } \Phi_{p} \in[0, k]
\end{aligned}
$$

And the final form of discretized equation is:

$$
\sum_{i} A_{i} \Phi_{i}+S_{\Phi}^{\prime}+S_{d c}=A_{p} . \Phi_{p} \quad i: E, W, N, S
$$

\subsubsection{Solution Algorithm}

The technique used to obtain the field of velocity and pressure in this research is PISO algorithm. This algorithm has 1 stage of prediction and 2 correction steps to calculate the velocity-pressure field for transient compressible flows and also Crank-Nicolson scheme [21] is employed for discretization of time derivative with second order accuracy.

\subsection{Solid Equations}

In the present research, motion equations in two degree of freedom have been used for aeroelastic modeling in pitch plunge system. The equations of motion are:

$$
[\mathrm{M}]\{\ddot{\mathrm{X}}\}+[\mathrm{D}]\{\dot{\mathrm{X}}\}+[\mathrm{K}]\{\mathrm{X}\}=\{\mathrm{F}(\mathrm{t})\}
$$

The two-dimensional motion equations are obtained from the Lagrangian equations which are combined with the aerodynamic equations. In this study the equations of motion in two cases were studied. In the first case the forced airfoil oscillation was studied under aerodynamic forces that the angle of attack is varied as a function of non-dimensional time $\mathrm{t}$ as:

$$
\theta=\theta_{0}+\theta_{\max } \sin (2 \kappa t)
$$

In the second case free airfoil oscillation was studied under aerodynamic forces and only damping under the influence of fluid viscosity is achieved and no structural damping is considered. The equations under these conditions are given in the form below. In these test cases stiffness centers are located in the middle of cord.

$$
\left\{\begin{array}{l}
F_{L} \cos (\theta)+F_{D} \sin (\theta)=K_{S} \cdot X \\
F_{L} \cos (\theta)^{c} / 4+F_{D} \sin (\theta)^{c} / 4+M_{c / 4}=K_{\theta} \cdot \theta_{0}
\end{array}\right.
$$

By solving these equations in each time step airfoil pitch angle and vertical displacement is obtained.

\section{Boundary Conditions}

Boundary conditions in this research include:

1: velocity inlet at input boundary

2: pressure outlet at the exit boundary

3: far-field boundary on the upper and lower boundaries

Also the airfoil distance is $5 \mathrm{C}$ from the inlet and $15 \mathrm{C}$ from the exit. Here the wall functions are used to calculate the boundary layer velocity [22]. The airfoil distance from the boundaries is set, to have the least effect from the boundaries or the independence of the computing area is observed.

\section{FSI Solution}

At the fluid-structure interaction, first Navier Stokes equations are solved around the airfoil and gained pressure and viscosity forces on the airfoil surface then they are used as inputs of the structure equations. Then by solving the structural equations vertical displacement and pitch angle are obtained at each time step. In this simulation because the mesh is constant even with large time steps or large vertical displacement the solution does not diverge. This problem is very important in the dynamic mesh because by increasing the deformation, the mesh is distorted in most cases and solution is diverged. FSI solution algorithm is given below.

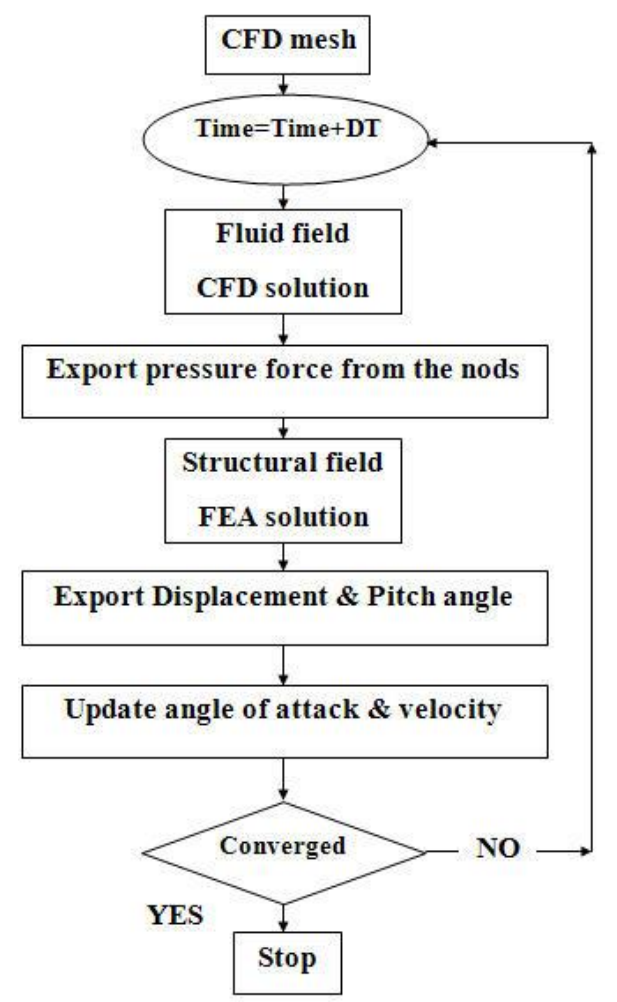

Fig2. FSI solution algorithm 


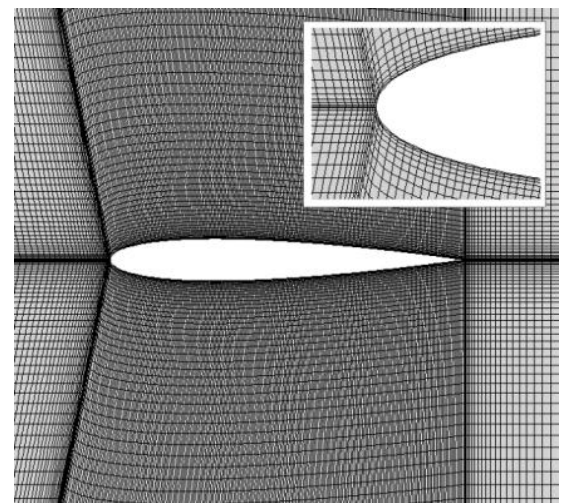

Fig3. Computational grid

\section{Results and Discussion}

First the forced airfoil oscillation was reviewed during the transonic flow in Mach 0.755.For this purpose first of all independence from the grid has been achieved.

The grid sizing is obtained by doing several different trials. The result of the independence of the computational grid is presented in Fig.4.As can be seen to simulate the flow, a grid with 115960 numbers of elements is utilized. After independence mesh is achieved, the validation of the solving method should be compared with valid laboratory results [23].For this purpose, an airfoil with NACA 0012 section which undergoing forced sinusoidal pitching in flow at a Mach 0.755 is used. Where $\kappa=0.0814$ is the reduced frequency and the primal and amplitude angles of attack are respectively $\theta_{0}=0.016^{\circ}$ and $\theta_{\max }=2.51^{\circ}$. As can be seen the numerical solution results in good agreement with experimental data Fig.5 [23].

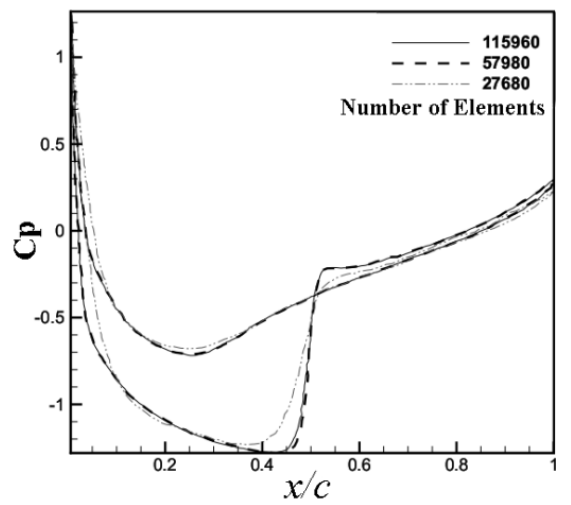

Fig4.The effect of grid sizing on pressure distribution

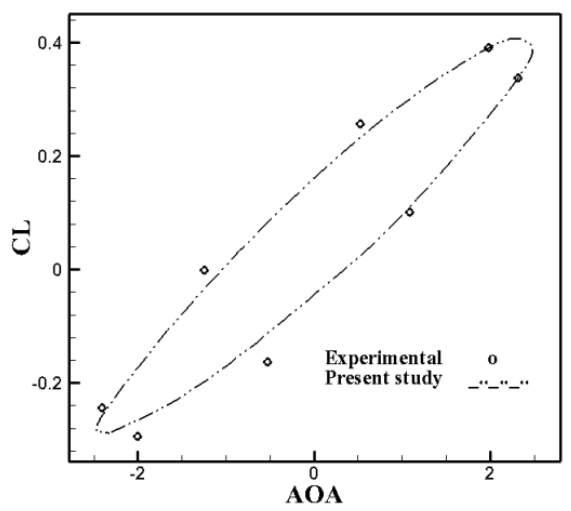

Fig5.Lift coefficient versus angle of attack in the forced airfoil oscillation

As can be seen in the forced airfoil oscillation, the upper and lower limit of maximum lift coefficients is fixed and the results with passage of time are repeated cyclically Fig.5.

The FSI test case considered the transonic flow at $\mathrm{M}_{\infty}=$ 0.8 for all test cases. For this purpose first, steady solution is to be provided with an initial angle of attack around the airfoil that the initial angle of attack is 1 degree for all test cases. This steady solution produces the field of pressure and velocity and other relevant parameters. While the distribution of forces around the airfoil was achieved, you can leave airfoil and examine it in different conditions. It is clear that airfoil movements respond differently in different situations. For example with changing the initial amplitude will give different answers. In this research because the aim of the study is to investigate the motion of airfoil under different conditions, attention is paid to roughness and structural parameters. The meaning or the innovation point of these test cases is to provide a new method for the two-dimensional aeroelastic simulation and to reduce computational cost and convergence faster.

First, it is necessary to examine the effect of added mass in this simulation and then compare the results with the results of this method. For this purpose, free airfoil oscillation in Mach 0.85 has been simulated. As can be seen, since the air density is low, and the range of airfoil oscillations is small, when the effect of added mass is considered, there is no significant change in the lift and drag coefficients and vertical displacement airfoil also can ignore this effect in this simulation method [24].

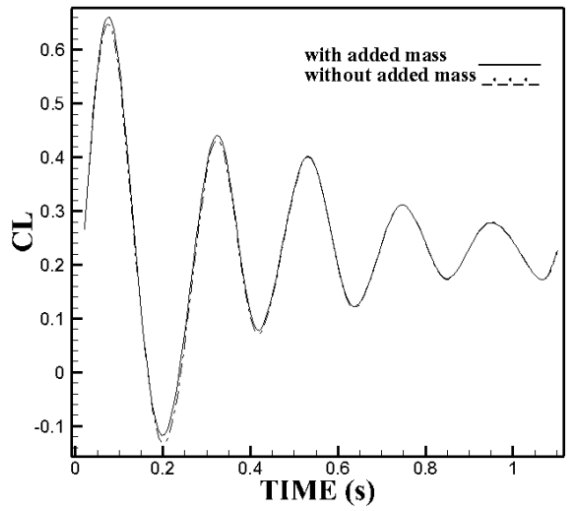

Fig6.Lift coefficient versus time

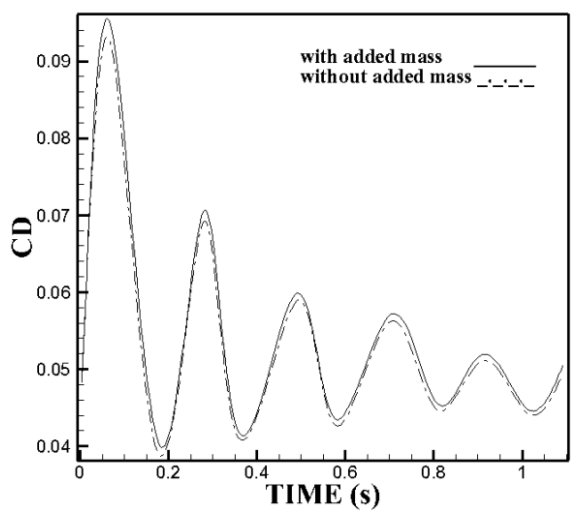

Fig7.Drag coefficient versus time 


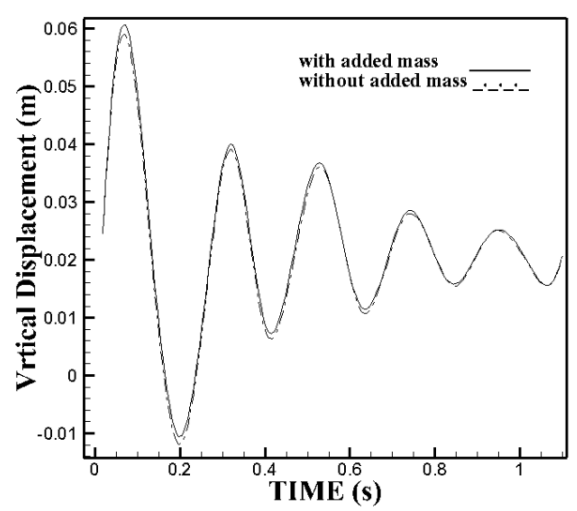

Fig8.Vertical displacement airfoil versus time

As can be seen, when the effect of the added mass is considered the variation of oscillation amplitudes in comparison with the present method is very slight and can be ignored.

In the first test case on airfoil NACA0012, the pitch $\left(K_{\theta}\right)$ and vertical stiffness $\left(K_{S}\right)$ values are:

$$
\left\{\begin{array}{l}
K_{s}=850470(N / M) \\
K_{\theta}=52500\left(N \cdot \frac{M}{r a d}\right)
\end{array}\right.
$$

Below changes of lift and drag coefficients are given at the angle of attack (Figs.9-10).

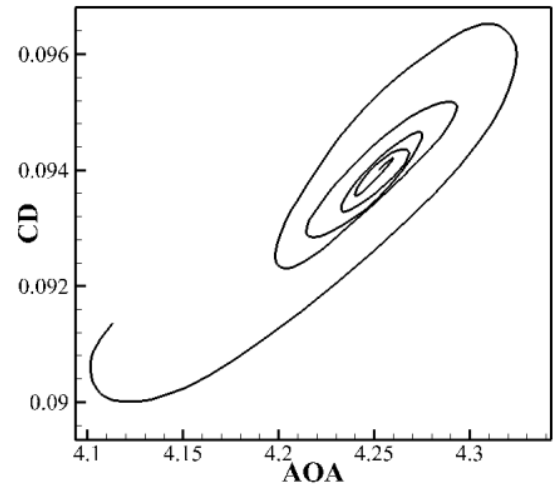

Fig9.Drag coefficient versus angle of attack

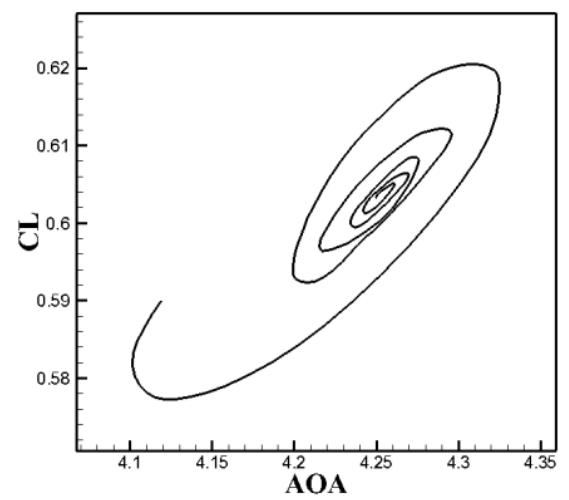

Fig10.Lift coefficient versus angle of attack

As you can see with the passage of time the range of lift and drag variations become smaller and converge to a steady value after several cycles. This state occurs when structural stiffness overcomes aerodynamic forces and causes domain variations to become smaller with time.

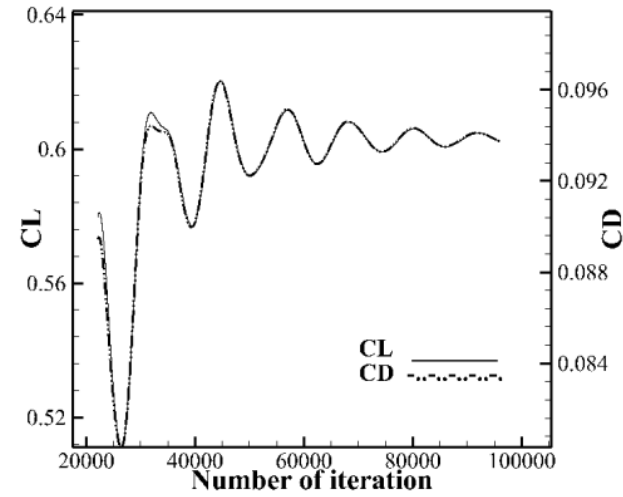

Fig11.Drag and Lift coefficients versus number of iteration

In Fig. 11 the behavior of the lift and drag coefficients are shown in time step. It can be seen here that when the value of lift coefficient reaches its maximum locally, the value of the drag coefficient is also maximal. In Figures 12 and 13 , the changes in the airfoil angle and the vertical displacement of the airfoil are shown in versus to time. It can be seen that when the lift factor reaches its maximum value, the airfoil angle and vertical displacement airfoil are also maximal.

It is also seen here that with the passage of time the airfoil oscillations are reduced. This shows that structural stiffness overcomes the aerodynamic forcesandcauses to converge to a constant value after several oscillations.

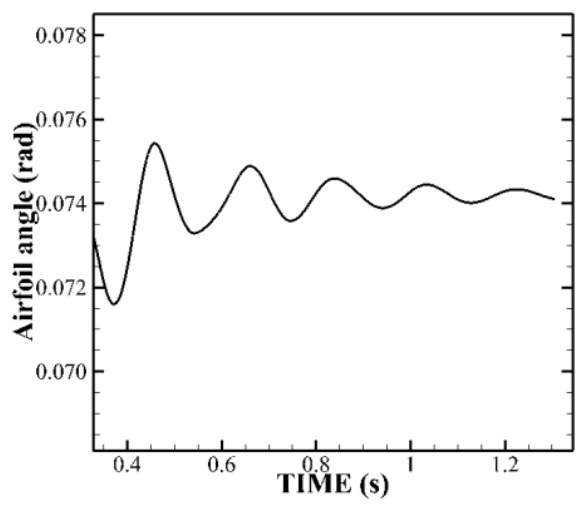

Fig12.Airfoil angle versus time

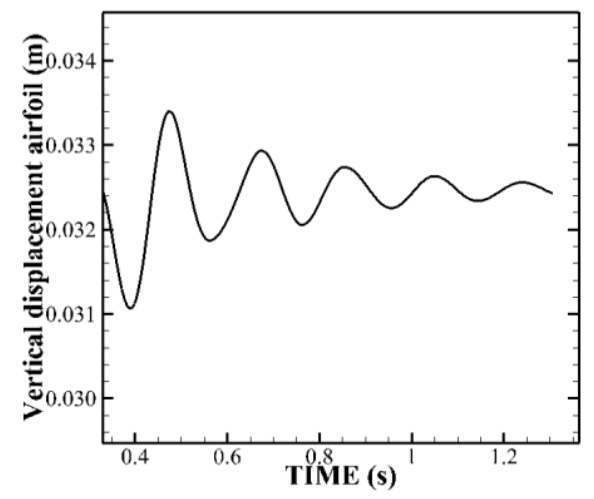

Fig13.Vertical displacement airfoil versus time 
In the second test case on airfoil NACA 0012 the pitch and vertical stiffness values are:

$$
\left\{\begin{array}{l}
K_{s}=1700940(N / M) \\
K_{\theta}=105000\left(N \cdot \frac{M}{r a d}\right)
\end{array}\right.
$$

In this test case the structural stiffness has doubled. Here the purpose of investigating is the behavior of airfoil in a specified Mach with increasing structural stiffness.

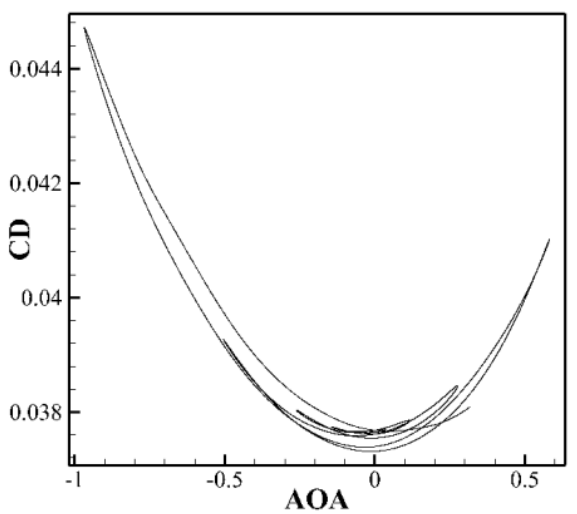

Fig14.Drag coefficient versus angle of attack

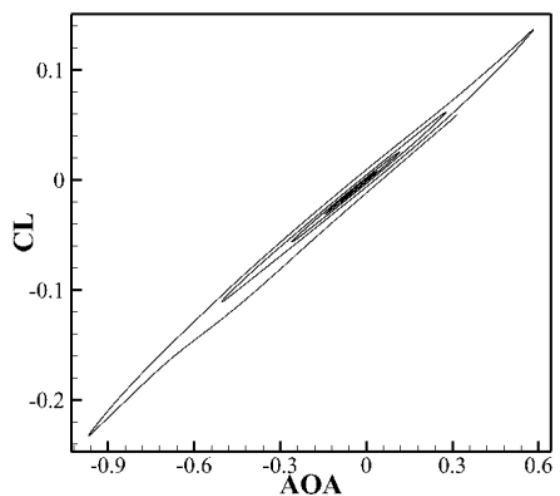

Fig15.Lift coefficient versus angle of attack

Changes of lift and drag coefficients are given on the angle of attack in figures 14 and 15 respectively. In this case since the structural stiffness is doubled, it causes the airfoil to fluctuate around its neutral axis and the lifting force in the upstroke and down stroke have positive and negative values. Also because the amplitude of airfoil angle is smaller, the amount of drag coefficient is lower than the previous one.

It is also observed here that when the value of the lift coefficient is locally maximal, the corresponding value of the drag coefficient is also in the maximum situation and as time passes the lift and drag coefficients are converted to steady values Fig. 16.

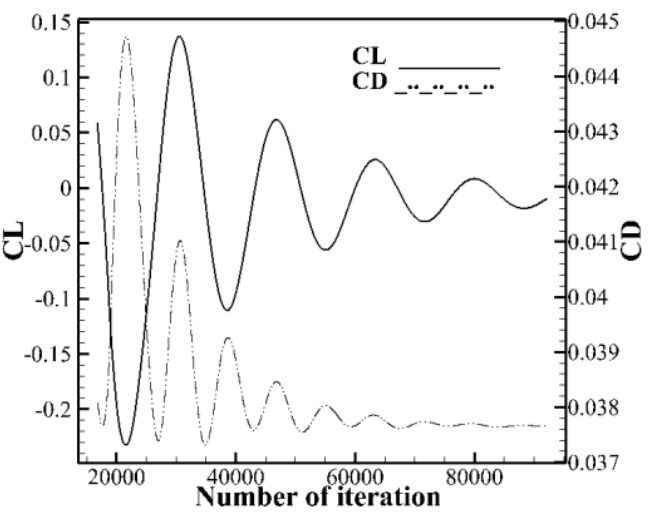

Fig16.Drag and Lift coefficients versus number of iteration

Figures 17 and 18 show that with increasing structural stiffness the vertical displacement and airfoil angle have been decreased. It is observed that after the release airfoil and with passage of time the amount of displacement converges to a steady state pose. It can also be seen that the number of oscillations, converge with higher rate to quantity of steady state relative to the previous case.

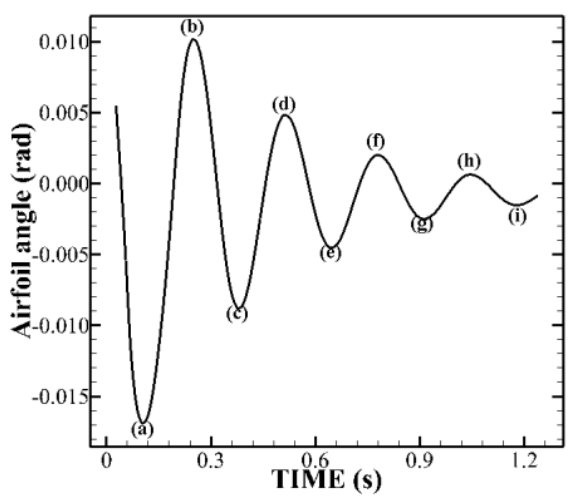

Fig17. Airfoil angle versus time

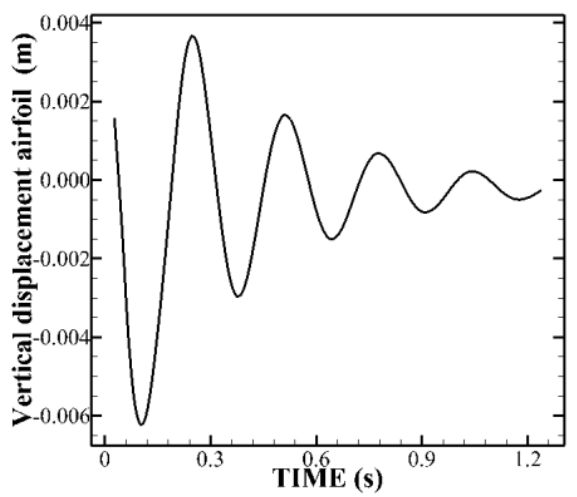

Fig18. Vertical displacement airfoil versus time

In the third test case on airfoil NACA 0012, the pitch and vertical stiffness values are:

$$
\left\{\begin{array}{l}
K_{s}=3401881.88(N / M) \\
K_{\theta}=210000 \quad\left(N \cdot \frac{M}{r a d}\right)
\end{array}\right.
$$

In this test case the structural stiffness has doubled relative to the second case. Initially changes of lift coefficient to angle of attack are given Fig. 19. 


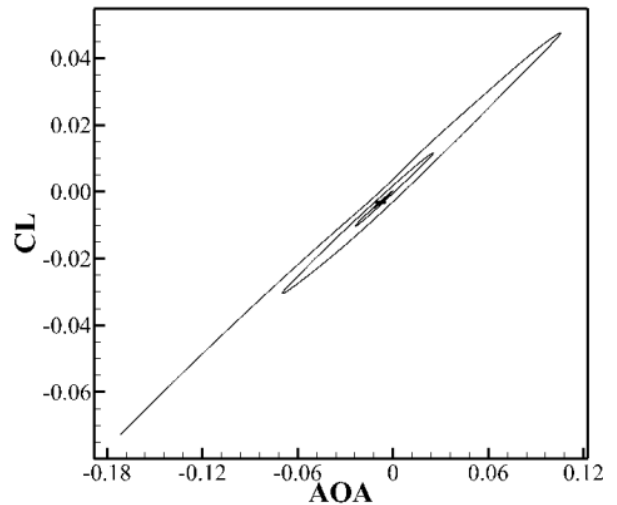

Fig19. Lift coefficient versus angle of attack

It is observed that the magnitude of the lift coefficient is lower than previous cases and the reason is that with increasing structural stiffness, the amount of angular variations has been decreased. Also the airfoil arrives sooner to its steady state pose.

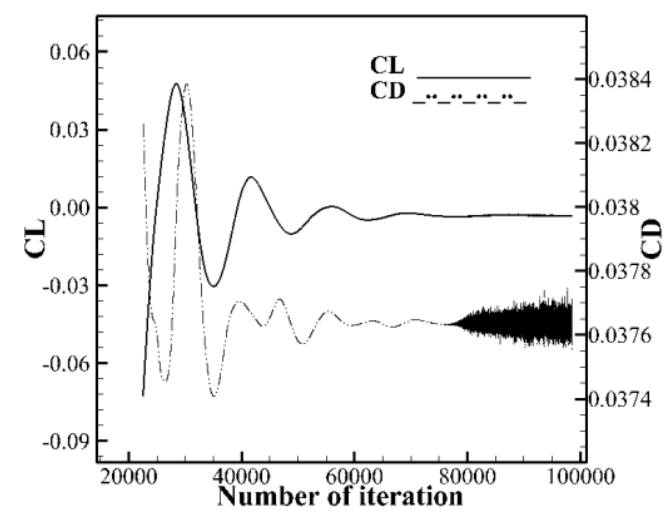

Fig20. Drag and Lift coefficients versus number of iteration

It is also clear that airfoil returns to its primary state after 2-3 fluctuations Fig 20. In this case the airfoil has come to its neutral state quickly it has had enough time to fluctuate around its neutral point so the drag coefficient has fluctuations around its neutral point. And it is clear that in this case fluctuations have a limit with the passage of time.

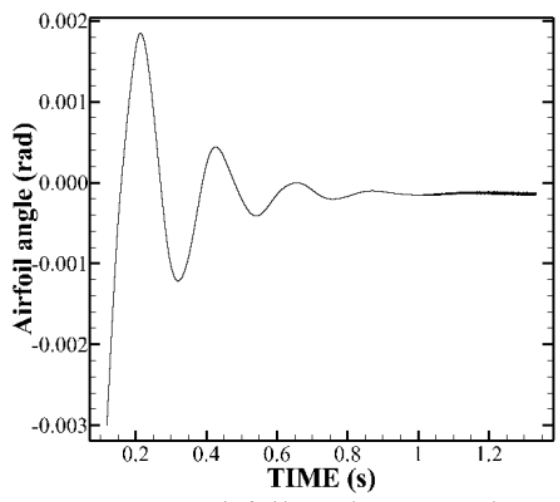

Fig21. Airfoil angle versus time

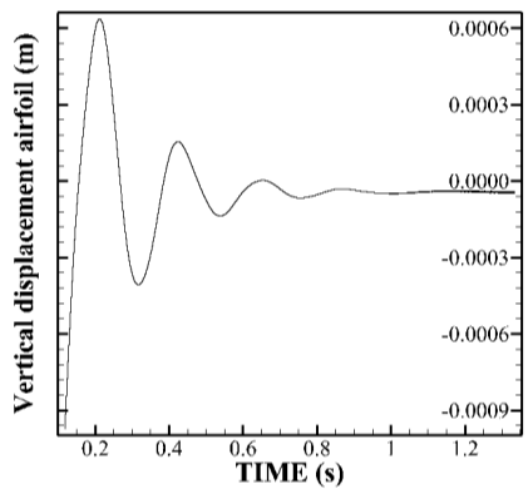

Fig22. Vertical displacement airfoil versus time

As shown in Figures 21 and 22 airfoil is almost constant after 1 second after release. It is also seen that the rate of decrease amplitude has increased relative to the previous cases.

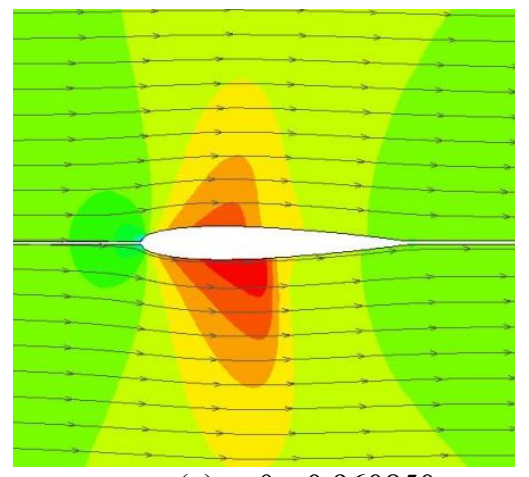

(a) : $\theta=-0.960850$

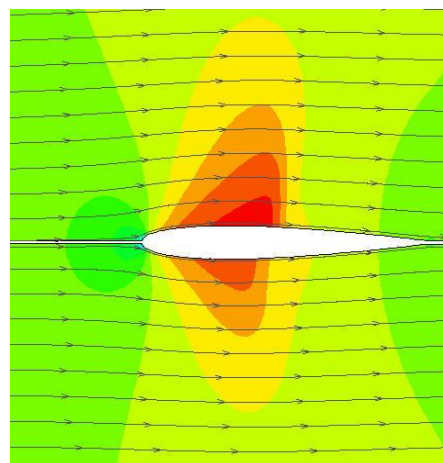

(b) $: \theta=0.578687$

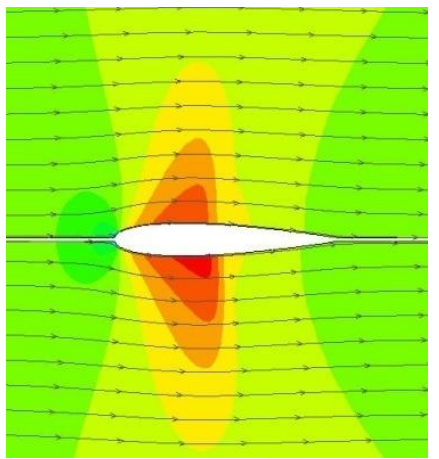

(c) $: \theta=-0.500021$ 


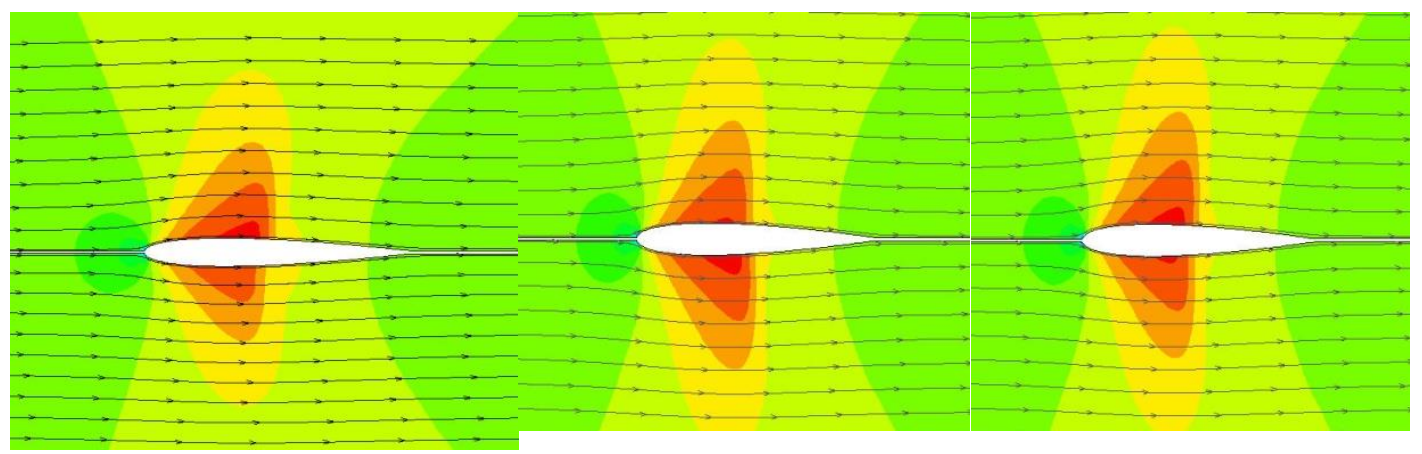

(d) $: \theta=0.274790$

(e) $: \theta=-0.258060$

(f) $: \theta=0.115680$

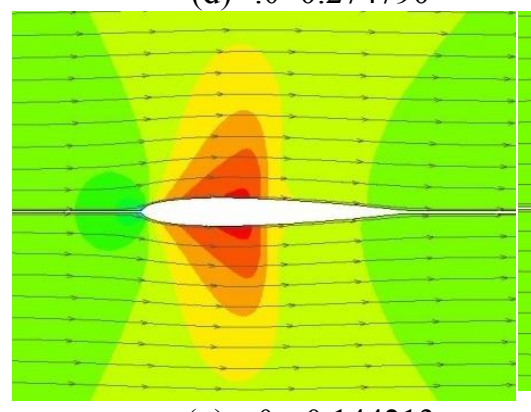

(g) $: \theta=-0.144213$

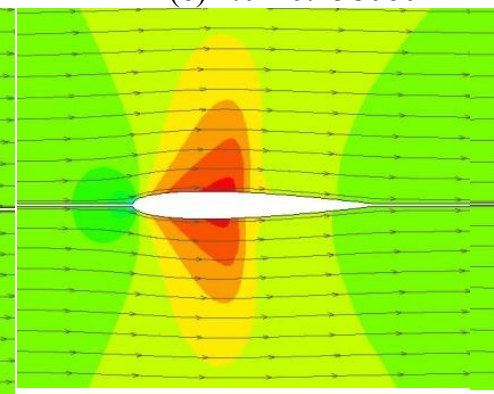

(h) $: \theta=0.034022$

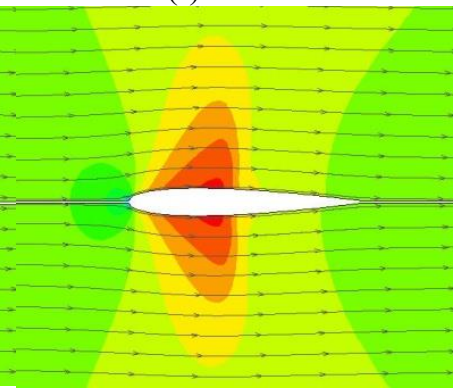

(i) $: \theta=-0.087662$

\section{MACH: $\quad 0 \quad 0.10 .20 .30 .40 .50 .60 .70 .80 .9 \quad 1 \quad 1.11 .2$}

Fig23.Mach contours around the airfoil at $\mathrm{M}=0.8, K_{S}=1700940(N / M), K_{\theta}=105000\left(N \cdot \frac{M}{\mathrm{rad}}\right)$

Figure 23 shows the distribution of the Mach coefficient contours corresponding to the positions shown in Fig14. As you can see the position of the shock wave in the upper and lower surface of the airfoil is distinct for different times or in other words for different angle of attack and by changing the angle of attack, the intensity of the shock wave changes. As time passes and decrease of the angle of attack, the position of the shock wave in upper and lower surface of the airfoil moves towards the trailing edge and has approximately identical values.

Here the results of the roughness effect on the aeroelastic are given. Due to the presence of roughness in the wall, the turbulence intensity near the rough wall is much greater than the smooth wall. In this simulation, the roughness height is 0.0002 meters.

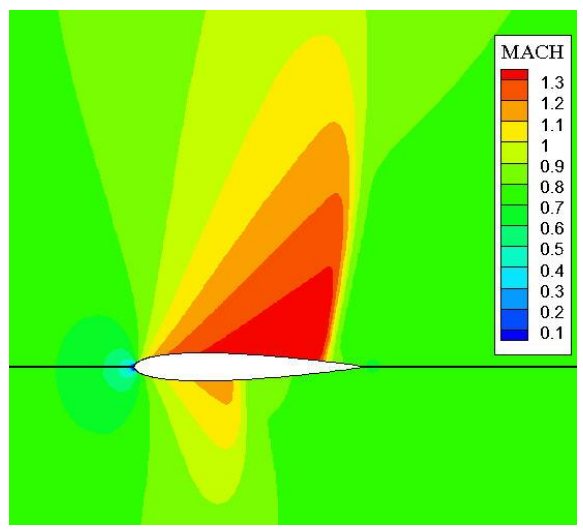

Fig24. Mach contours around the smooth airfoil

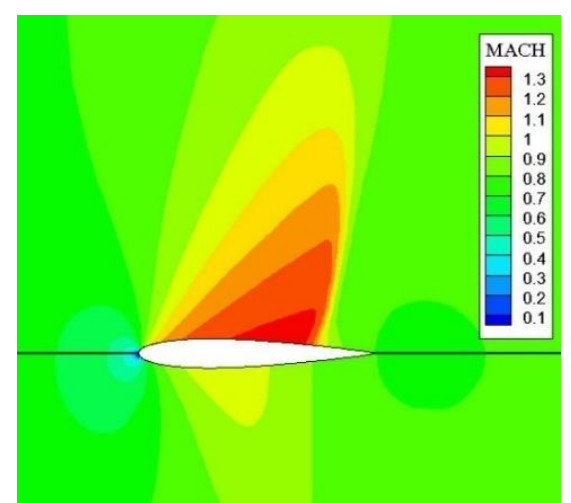

Fig25. Mach contours around the rough airfoil

In figures 24 and 25, Mach's contours around the oscillating airfoil are presented in two smooth and rough modes respectively.

As can be seen from the comparison of two smooth and rough surfaces it can be concluded that when roughness is applied on the surface, shock strength dropped and also the spot of the shock is moved to the leading edge.

Figures [26-27] show the distribution of the pressure coefficient on the smooth and rough surfaces of the oscillating airfoil respectively. It is also observed, in the rough surface the pressure distribution at the bottom surface is more uniform and there is no severe pressure gradient. 


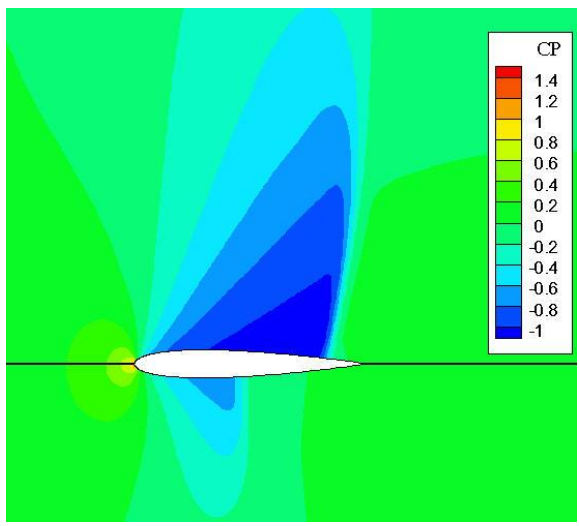

Fig26. Pressure coefficient contours around the smooth airfoil

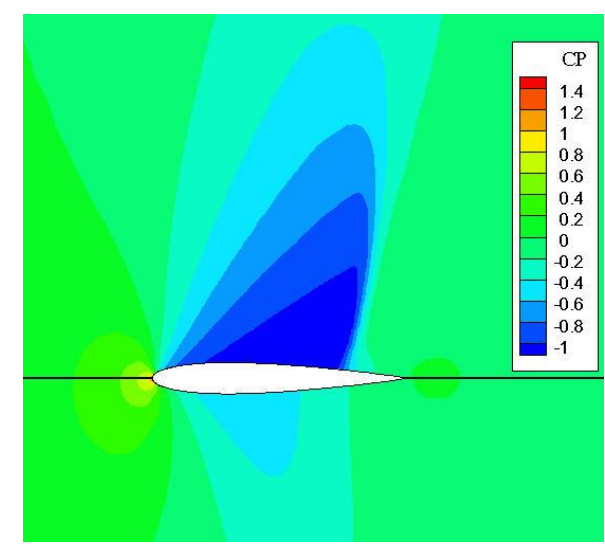

Fig27. Pressure coefficient contours around the rough airfoil

In Fig.28 the distribution of the pressure coefficient in two smooth and rough surfaces are compared. As can be seen on smooth surface the shock has occurred at both the upper and lower surfaces while on a rough surface, the shock has occurred only at upper surface. In this figure it is easy to see that shock's place in the rough airfoil has been relocate to the leading edge.

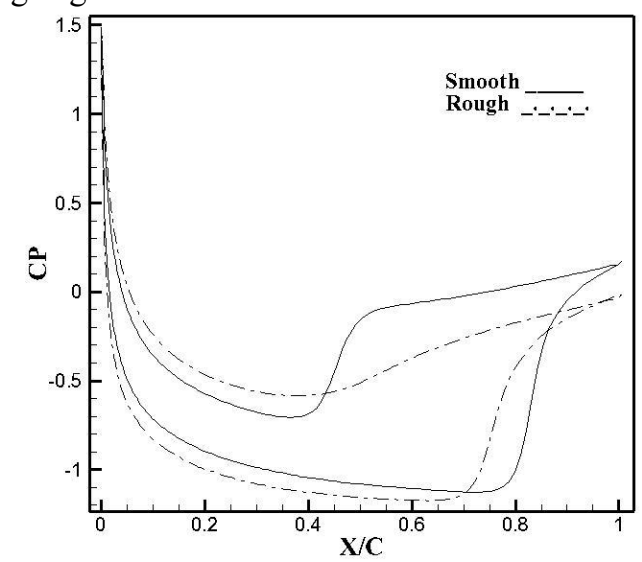

Fig28. Pressure coefficient distribution around the smooth and rough airfoils

In Figure 29 the distribution of wall shear stress were compared. When the surface is rough, it causes the turbulence intensity near the wall, the values of $u^{\prime}$ and $v^{\prime}$ and the shear stress of the rough wall are increased. Here it is also observed that when a shockwave occurs, the wall shear stress is suddenly dropped.

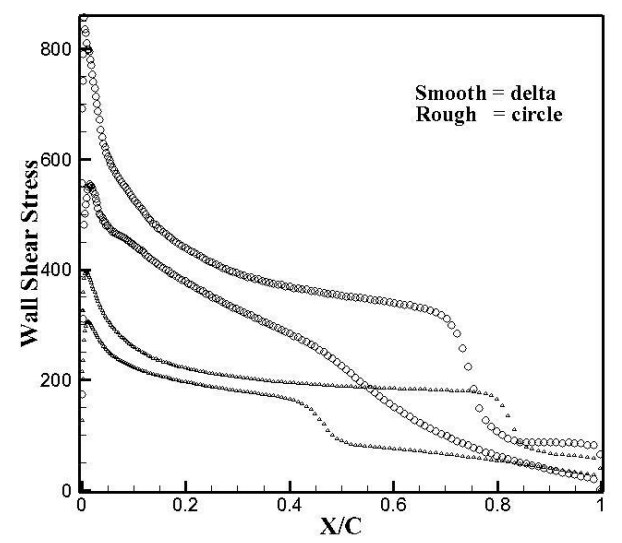

Fig29. Comparison of wall shear stress around the smooth and rough airfoils

In figure 30, the lift coefficient in two smooth and rough conditions is compared. It is observed that in a rough surface, the lift values are less than smooth surface, and the airfoil has reached a steady state earlier.

Figure 31 shows that at a rough surface, the amount of drag is much larger than the smooth surface because the shear stress of the smooth surface is less than the rough surface.

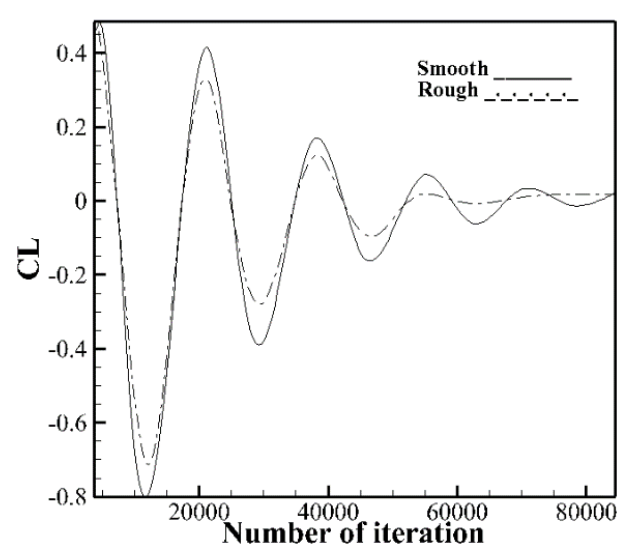

Fig30. Comparsion of lift coefficient versus number of iteration around the smooth and rough airfoils

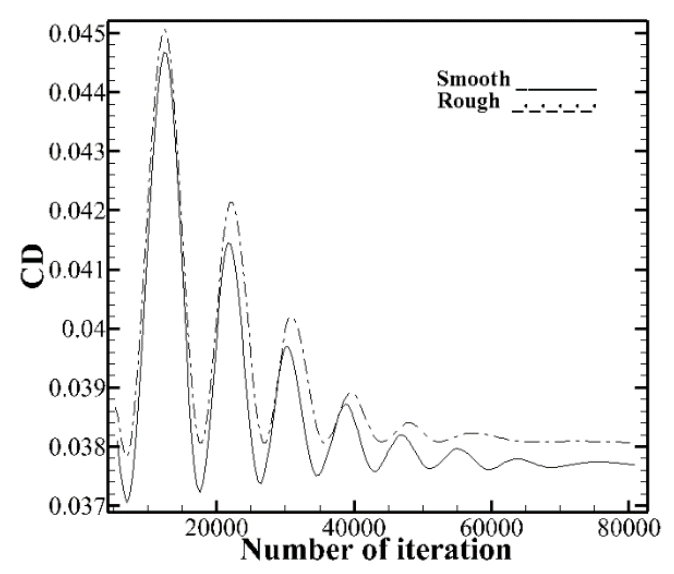


Fig31. Comparison of drag coefficient versus number of iteration around the smooth and rough airfoils

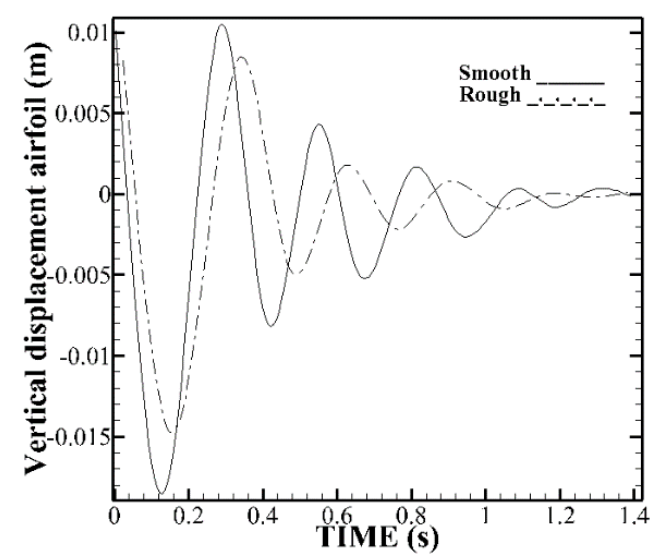

Fig32. Comparsion of vertical displacement around the smooth and rough airfoils

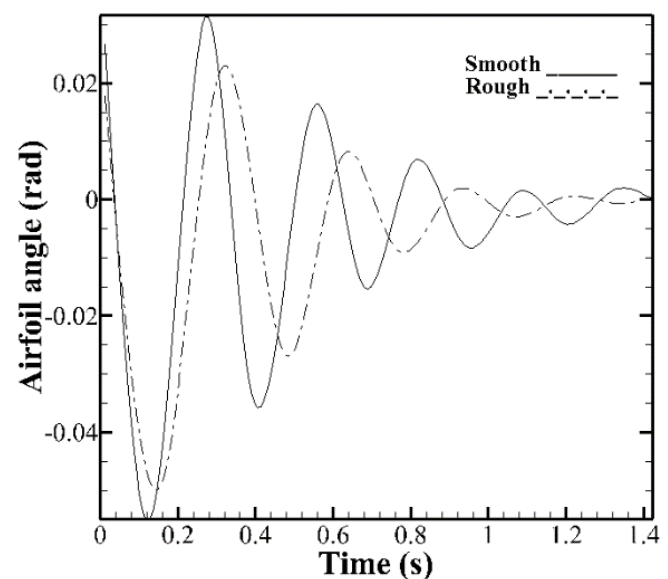

Fig33. Comparsion of airfoil angle around the smooth and rough airfoil

In figures 32 and 33 vertical displacement and airfoil angle are brought. Because in a rough surface, aerodynamic forces are smaller, structural stiffness will play a more effective role in the movement of airfoil and the rough airfoil will reach its steady state earlier, and it will have a smaller fluctuation range.

\section{CONCLUSION}

In this article the static mesh technique is used instead of a dynamic mesh that is most costly. Also to simulate Navier Stokes equations, a modified model for compressible flow and for computing convection term high order SBIC scheme based on discretization of Normalized Variable Diagramis used. The results of validation and simulation show that the extracted data has a desired accuracy and had good agreement to experimental results. Some principal points can be explained as follow.

- The new numerical method provides acceptable results of $2 \mathrm{D}$ aeroelastic.

- The concurrence between experimental data and numerical results are considerable.
- The present technique is considered to provide effective method in aeroelastic simulation in which the mesh is fixed and it does not distort.

- This simulation method is much simpler and less costly than other methods.

- The results show that with increasing structural stiffness the number of oscillations and drag coefficient decreases.

- This method takes less time to converge than other methods.

- The results show that with increasing structural stiffness, oscillations are eliminated more quickly.

- By applying surface roughness it causes the shock strength to drop and the shock'splace moves to the leading edge.

- Also the roughness causes the airfoil to reach a steady state earlier and the number of fluctuations is lower.

\begin{tabular}{|c|c|c|}
\hline \multicolumn{3}{|c|}{ Nomenclature } \\
\hline FSI & $=$ & Fluid-Structure Interaction \\
\hline$S B I C$ & $=$ & $\begin{array}{l}\text { Second and Blending Interpolation } \\
\text { Combined }\end{array}$ \\
\hline$N V D$ & $=$ & Normalized Variable Diagram \\
\hline$C F D$ & $=$ & Computational Fluid Dynamic \\
\hline SIMPLE & $=$ & $\begin{array}{l}\text { SemiImplicit Method For Pressure Linked } \\
\text { Equation }\end{array}$ \\
\hline$A O A$ & $=$ & Angle of Attack (in degree) \\
\hline $\mathrm{K}$ & $=$ & SBIC parameter \\
\hline$R e$ & $=$ & Reynolds number \\
\hline$c$ & $=$ & Chord Length \\
\hline$\tilde{a}$ & $=$ & Cell Face Area \\
\hline$C_{\mu}, C_{1}, C_{2}$ & $=$ & Empirical coefficients \\
\hline$u_{\tau}$ & $=$ & Friction velocity \\
\hline$\kappa$ & $=$ & Von karman constant \\
\hline$\tau_{w}$ & $=$ & Wall shear stress \\
\hline$h_{s}$ & $=$ & Roughness height \\
\hline$F$ & $=$ & Mass flux \\
\hline I & $=$ & Flux \\
\hline$k$ & $=$ & Kinetic Energy of Turbulence \\
\hline$M_{\infty}$ & $=$ & Free stream Mach number \\
\hline$q$ & $=$ & Scalar flux vector \\
\hline$T$ & $=$ & Stress tensor \\
\hline$u, v$ & $=$ & Velocity components in $\mathrm{X}$ and $\mathrm{Y}$ directions \\
\hline$\Gamma$ & $=$ & Diffusivity coefficient \\
\hline$\Gamma^{t}$ & $=$ & Turbulent diffusivity coefficient \\
\hline$\delta v$ & $=$ & Cell volume \\
\hline$\varepsilon$ & $=$ & Volumetric rate of dissipation \\
\hline$\mu$ & $=$ & Dynamic viscosity \\
\hline$\mu_{t}$ & $=$ & Turbulent viscosity \\
\hline$\rho$ & $=$ & Density \\
\hline$\sigma_{k}$ & $=$ & $\begin{array}{l}\text { Turbulent Prandtl number for turbulent } \\
\text { kinetic energy }\end{array}$ \\
\hline$\sigma_{\varepsilon}$ & $=$ & $\begin{array}{l}\text { Turbulent Prandtl number for dissipation } \\
\text { rate }\end{array}$ \\
\hline$\phi$ & $=$ & Scalar quantity \\
\hline
\end{tabular}




$\begin{array}{ll}\widetilde{\phi} & =\text { Normalized scalar quantity } \\ A, D & =\text { Finite difference coefficients } \\ \kappa & =\text { Reduced frequency } \\ X & =\text { Vertical displacement } \\ K_{0} & =\text { Spring matrix } \\ D & =\text { Dynamic damper matrix } \\ M & =\text { Mass of the airfoil } \\ K_{s} & =\text { Plunging spring coefficient } \\ K_{\theta} & =\text { Pitching spring coefficient } \\ M c / 4 & =\text { Moment about the aerodynamiccentre } \\ F_{L} & =\text { Lift force } \\ F_{D} & =\text { Drag force } \\ \theta & =\text { Pitch angle } \\ f & =\text { Physical frequency } \\ \omega_{a} & =\text { Circular frequency }\end{array}$

\section{References}

[1]Y. Fung, An introduction to the theory of aeroelasticity. Mineola, N.Y.: Dover Publications, 2008.

[2] S. Lapointe and G. Dumas, "Numerical Simulations of Self-Sustained Pitch-HeaveOscillations of a NACA0012 Airfoil", Renewable Energy, 42, 66-76, 2012

[3] A. G. B. Mowat, A. G. Malan, L. H. Van Zyl and J. P.Meyer ,"A hybrid finite volume ROM approach to nonlinear aerospace fluid-structure interaction modelling ",IFASD-2011-008

[4] M. Khairil and H. M. Zorkipli, "Simulation of aeroelastic system with aerodynamic nonlinearity ",Proceedings of the International Conference on Vibration, Sound and System Dynamics Penang, 2 August 2017

[5]R. Unger, M. C. Haupt and P. Horst, "Structural Design and Aeroelastic Analysis of an Oscillating Airfoil for Flapping Wing Propulsion", Aerospace Sciences Meeting and Exhibit 7 - 10 January 2008, Reno, Nevada, AIAA 2008-306

[6] C. Riso, G. Riccardi and F.Mastroddi, "Nonlinear aeroelastic modeling via conformal mapping and vortex method for a flat-plate airfoil in arbitrary motion", Journal of Fluids and Structures, vol. 62, pp. 230-251, 2016

[7] W. Medjroubi, B. Stoevesandt, B. Carmo and J. Peinke,"High-order numerical simulations of the flow around a heaving airfoil", Computers \& Fluids, vol.51, no. 1 , pp. $68-84,2011$

[8] W. Yuan, B. Wang, and D. Poirel, "Numerical Simulations of Self-Sustained Aeroelastic Oscillations at Low Reynolds Numbers," 28th Congress of the International Council of the Aeronautical Sciences, Brisbane, Australia, Sep. 2012

[9] N. Banavara and D. Dimitrov,"Prediction of Transonic Flutter Behavior of a Supercritical Airfoil Using Reduced Order Methods", Notes on Numerical Fluid Mechanics and Multidisciplinary Design, pp. 365-373, 2014

[10] J. Akkala, A. Eslam Panah, B. Goodman and J. Buchholz, "Vortex Dynamics and Performance of a Flexible Plunging Airfoil", 51st AIAA Aerospace
Sciences Meeting including the New Horizons Forum and Aerospace Exposition, 2013

[11] N. Razak, T. Andrianne and G. Dimitriadis,"Flutter and Stall Flutter of a Rectangular Wing in a Wind Tunnel", AIAA Journal, vol. 49, no. 10, pp. 2258-2271, 2011

[12] W.Walker and M. Patil,"Unsteady Aerodynamics of Deformable Thin Airfoils", Journal of Aircraft, vol.51, no. 6, pp. 1673-1680, 2014

[13] M. Williams,"Linearization of Unsteady Transonic Flows Containing Shocks", AIAA Journal, vol. 17, no. 4, pp. 394-397, 1979

[14]Tao Bai, Jingyuan Liu, Weihao Zhang, Zhengping Zou "Effect of surface roughness on the aerodynamicperformance of turbine blade cascade" Propulsion and Power Research 2014

[15]Abdelhamid Bouhelal, Arezki Smaili, Christian Masson, Ouahiba Guerri"Effects of Surface Roughness on Aerodynamic Performance of Horizontal Axis Wind Turbines "The 25 Annual Conference of the Computational Fluid Dynamics Society of Canada 2017

[16] Ece Sagol, Marcelo Reggio, Adrian Ilinca "Issues concerning roughness on wind turbine blades" Renewable and Sustainable Energy Reviews 23 (2013) Elsevier 514-525

[17] Beatriz Mendez, Arturo Munoz and Xabier Munduate "Study of distributed roughness effect over windturbine airfoils performance using CFD" AIAA SciTech 2015

[18] Yang, Z.Y. Chin, S.B., and Swithenbank, J., On the Modeling of the k-Equation for compressible Flow, 7th International Symposium on Numerical Methods in Laminar and Turbulent Flow, Stanford, CA, July 1991

[19] Narayam, J.R, and Sekar, B., 1991, Computational of Turbulent High Speed Mixing Layers Using a Twoequation Turbulence Model, CFD symposium on aeropropulsion, NASA CP-3078, Jan.

[20] Rubin S.G. and Khosla P.K., Polynomial Interpolation Method for Viscous Flow Calculations, J. Comput. Phys., Vol.27, pp.153-168, 1982

[21] Issa, R.I., 1986, Solution of the implicitly discretised fluid flow equations by operator-splitting, Journal of Computational Physics 62, pp.40-65.

[22]Launder, B. E. and Spalding, D. B., The Numerical Computation of Turbulent Flows, Computer Methods in Applied Mechanics and Engineering, Vol. 3., pp. 269, 1974

[23] Landon, R.H., 1982, NACA 0012 Oscillating and Transient Pitching, in AGARD Report.

[24]Alexandr I. Korotkin,Added Masses of Ship Structures, FLUID MECHANICS AND ITS APPLICATIONS, 2009 Springer Science

$* * *$

\section{Creative Commons Attribution License 4.0 (Attribution 4.0 International, CC BY 4.0)}

This article is published under the terms of the Creative Commons Attribution License 4.0 https://creativecommons.org/licenses/by/4.0/deed.en_US 